\title{
The Protective Effects of a Modified Xiaohua Funing Decoction against Acute Liver Failure in Mice Induced by D-Gal and LPS
}

\author{
Jindong Zhao $i$, ${ }^{1,2}$ Lili Liu, ${ }^{3}$ Ling Xin, ${ }^{4}$ Yunxia Lu, ${ }^{5}$ Xiaojun Yang, ${ }^{3}$ Yong Hou, ${ }^{3}$ Mei Shi, ${ }^{3}$ \\ Sha Han, ${ }^{3}$ Hao Zhou, Yonghua Liu, ${ }^{3}$ Zhaohui Fang $\mathbb{D}^{2},{ }^{2}$ Yan Li $\mathbb{D}^{3},{ }^{3}$ and Guoliang Zhang $\mathbb{D}^{3}$ \\ ${ }^{1}$ Graduate School, Anhui University of Chinese Medicine, Hefei 230012, China \\ ${ }^{2}$ Department of Endocrinology, The First Affiliated Hospital of Anhui University of Chinese Medicine, Hefei 230031, China \\ ${ }^{3}$ Department of Infectious Disease, The First Affiliated Hospital of Anhui University of Chinese Medicine, Hefei 230031, China \\ ${ }^{4}$ Department of Information, The First Affiliated Hospital of Anhui University of Chinese Medicine, Hefei 230031, China \\ ${ }^{5}$ Department of Biochemistry and Molecular Biology, Anhui Medical University, Hefei 230032, China
}

Correspondence should be addressed to Zhaohui Fang; fangzhaohui1111@163.com, Yan Li; liyanzjd@163.com, and Guoliang Zhang; zhangguoliang61@qq.com

Received 11 December 2020; Revised 25 March 2021; Accepted 23 April 2021; Published 13 January 2022

Academic Editor: José Roberto Santin

Copyright (C) 2022 Jindong Zhao et al. This is an open access article distributed under the Creative Commons Attribution License, which permits unrestricted use, distribution, and reproduction in any medium, provided the original work is properly cited.

\begin{abstract}
Objective. The aim of this study was to evaluate the effects of a modified Xiaohua Funing decoction (Xfd) on acute liver failure (ALF) and determine whether the protective mechanisms are related to alterations in the gut microbiota. Methods. An animal model of ALF was induced by intraperitoneal injection of D-galactosamine (D-Gal, $0.5 \mathrm{~g} / \mathrm{kg}$ ) and lipopolysaccharide (LPS, $100 \mu \mathrm{g} / \mathrm{kg}$ ). Male BALB/c mice were randomly divided into the following 4 groups: the control group (saline, Con), model group (D-Gal/LPS, Mod), silymarin pretreatment group $(200 \mathrm{mg} / \mathrm{kg}$, Sil), and modified Xfd pretreatment group $(650 \mathrm{mg} / \mathrm{kg}, \mathrm{Xfd})$. The Sil and Xfd groups received the respective intervention orally for 14 days and $2 \mathrm{~h}$ before D-Gal/LPS treatment. The liver injury markers included alanine aminotransferase (ALT) and aspartate aminotransferase (AST) levels and liver histology. 16S rRNA gene sequencing was performed to assess the effects on the caecum content. Results. D-Gal/LPS treatment caused severe ALF, illustrating that the ALF model was successfully established. The administration of Sil and Xfd greatly reduced the serum ALT and AST levels and improved the pathological signs of liver injury. However, no significant difference was found between the two groups. In contrast to the Mod group, the Sil and Xfd groups showed a shift toward the Con group in terms of the gut microbiota structure. The abundances of Firmicutes and Bacteroidetes and the Bacteroidetes/Firmicutes ratio in the Mod group significantly differed from those in the Con group. The Sil and Xfd groups showed restoration of the disordered microbiota. Significantly increased relative abundances of Lachnospiraceae_NK4A136_group and Candidatus_Saccharimonas and a markedly decreased Muribaculaceae abundance were found in the Sil and Xfd mice compared with those in the Mod mice $(P<0.01, P<0.05)$. Interestingly, a negative correlation was observed between the abundances of the gut microbiota constituents, specifically Clostridia_UCG-014, and ALT and AST levels. Conclusion. In summary, our results indicate that Xfd may protect the liver and modify the gut microbiota in ALF mice.
\end{abstract}

\section{Introduction}

Acute liver failure (ALF) caused by extensive hepatocyte necrosis is a fatal clinical syndrome and has been well characterized. However, the underlying mechanisms are not fully understood [1]. Studies have demonstrated that alterations in the gut microbiota play a crucial role in the pathogenesis of liver diseases $[2,3]$. During the early stage of ALF, the systemic and intestinal immune systems are suppressed, which leads to a rapid loss of normal liver function, multiple organ failure, and even death [4]. Despite advances in medical therapies, effective clinical ALF treatment is lacking $[5,6]$.

In China, Chinese herbal medicine (CHM) therapy for ALF has been widely used. Several clinical trials have shown that 
CHM can provide ALF patients with important alternative and complementary therapy benefits, such as improvement in systemic and liver recovery, regulation of inflammatory mediators, and induction of antiapoptotic genes [7-9]. Modified Xiaohua Funing decoction (Xfd) was developed by a nationally celebrated expert in traditional Chinese medicine (TCM), Jingshi Xu. Here, we report that Xfd includes Bambusae Caulis in Taenias, Bupleuri Radix, Pinelliae Rhizoma Praeparatum cum Zingibere et Alumine, Aurantii Fructus, Setariae Fructus Germinatus, Paeoniae Radix Alba, Schisandrae Fructus Chinensis, Salviae Miltiorrhizae Radix et Rhizoma, Glehniae Radix, Citri Reticulatae Pericarpium, and Mume Fructus. For over five decades, Xfd has been known to have a significant impact on liver diseases. Our previous clinical studies suggested that Xfd improved liver function and other symptoms, such as alanine aminotransferase (ALT), aspartate aminotransferase (AST), total protein, total bilirubin, and alkaline phosphatase levels; lateral thorax pain; stomach pain; loss of appetite; abdominal distension; loose stools; and jaundice. Xfd not only is effective but also has no other adverse effects, such as induction of kidney failure and diarrhea $[10,11]$. Several protective mechanisms of Xfd have been investigated based on the inflammatory response and the extracellular regulated protein kinase (ERK) signaling pathway [12].

The classical liver injury model induced by D-galactosamine (D-Gal)/lipopolysaccharide (LPS) has been used to evaluate abnormal liver function, regulation of metabolic disorders, and variations in the gut microbiota $[13,14]$. This mouse experimental model mimics clinically observed ALF [15]. The present study aims to investigate the effects of Xfd on gut microbiota and liver function in the context of ALF.

\section{Materials and Methods}

2.1. Materials and Reagents. Xfd contains 11 medicinal components, namely, Bambusae Caulis in Taenias, Bupleuri Radix, Pinelliae Rhizoma Praeparatum cum Zingibere et Alumine, Aurantii Fructus, Setariae Fructus Germinatus, Paeoniae Radix Alba, Schisandrae Fructus Chinensis, Salviae Miltiorrhizae Radix et Rhizoma, Glehniae Radix, Citri Reticulatae Pericarpium, and Mume Fructus, at a ratio of $15: 10: 10: 15: 30$ : $20: 10: 12: 15: 12: 10$. Xfd has been authenticated and standardized based on marker compounds according to the Chinese Pharmacopoeia 2020 [16]. A dose of $159 \mathrm{~g}$ of herbs was decocted with boiling water and concentrated with a vacuum rotary evaporator until crude aqueous extract. Silymarin was purchased from Macklin Biochemical Co., Inc. (Shanghai, China). Its purity was greater than $80 \%$. LPS (from Escherichia coli, 055:B5) and D-Gal were obtained from Sigma-Aldrich Chemical Co. (St. Louis, MO, USA). ALT and AST assay kits were provided by Nanjing Jiancheng Bioengineering Institute (Nanjing, China). A paraformaldehyde solution, acetonitrile, and formic acid were acquired from Macklin Biochemical Co., Inc. (Shanghai, China).

2.2. Animals and Treatment. Thirty-two male BALB/c mice (7 weeks old, 20-22 g) were obtained from the Shandong Laboratory Animal Center (Jinan, China; certificate number
SCXK-20190003). The animals were acclimated in a specific pathogen-free animal laboratory at $22 \pm 2^{\circ} \mathrm{C}$ with $50-70 \%$ relative humidity under a $12 / 12 \mathrm{~h}$ light/dark cycle. The mice were fed standard laboratory chow and given water ad libitum.

The mice were randomly divided into 4 groups $(n=8)$. The control group (Con) and model group (Mod) received the same volume of saline intragastrically once daily, the pretreatment silymarin group (Sil) received $200 \mathrm{mg} / \mathrm{kg}$ silymarin intragastrically once daily, and the pretreatment Xfd group (Xfd) received $650 \mathrm{mg} / \mathrm{kg}$ Xfd intragastrically once daily. The pretreatment course was 14 days. The bodyweight of the mice was measured every week.

Two hours after the last intragastric treatment, the mice in the Mod, Sil, and Xfd groups were treated with D-Gal $(0.5 \mathrm{~g} / \mathrm{kg}) / \mathrm{LPS}(100 \mu \mathrm{g} / \mathrm{kg})$ via intraperitoneal injection to induce ALF; D-Gal and LPS were freshly dissolved in saline. The mice in the control group were treated with the same volume of saline and with the same procedures. The experimental design and mouse handling procedures were in accordance with the guidelines for the care and use of laboratory animals.

2.3. Sample Collection and Analysis. Eight hours after treatment, the mice were killed by retroorbital bleeding, and blood samples, liver tissues, and cecal contents were collected.

2.3.1. Assessment of $A L F$. The blood was centrifuged for $10 \mathrm{~min}$ at $14,000 \mathrm{rpm}$ at $4^{\circ} \mathrm{C}$. The supernatant was stored at $-80^{\circ} \mathrm{C}$ until biochemical analysis. The ALT and AST levels were determined using an automatic biochemical analyzer (AU 7600, HITACHI, Japan).

2.3.2. Histological Evaluation. The liver tissue was weighed, washed with saline, and immediately placed into a tube containing 4\% paraformaldehyde solution. The liver tissues were washed with phosphate-buffered saline (PBS), embedded in paraffin wax, sliced into $5 \mu \mathrm{m}$ sections, and stained with hematoxylin-eosin ( $\mathrm{HE}$ ). The sections were analyzed under a Zeiss Axioskop 2 Plus upright light microscope equipped with a camera (Zeiss, Oberkochen, Germany).

2.3.3. $16 S$ rRNA Sequencing. The cecal contents were collected in sterile tubes and stored at $-80^{\circ} \mathrm{C}$ until gut microbiota analysis. Total bacterial DNA was extracted using a TIANamp Stool DNA Kit (Tiangen Biotech Co., Ltd., Beijing, China) following the manufacturer's instructions. The 16S rRNA gene in the DNA samples was amplified using the conventional barcoded universal bacterial primers F338/ R806 targeting the V3-V4 region, which were selected to analyze the taxonomic composition of the gut microbiota. The concentrations of the PCR products were determined using an Illumina HiSeq platform (Illumina, SD, USA) for paired-end reads with $2 \times 300 \mathrm{bp}$ ( $468 \mathrm{bp}$ ) sequencing. The trimmed sequences were uploaded to the Quantitative Insights Into Microbial Ecology (QIIME, v1.8.0) and $R$ 
packages (v3.3.1) $[17,18]$. The acquired data were produced by Majorbio Bio-pharm Technology Co., Ltd. (Shanghai, China).

The number of shared and unique operational taxonomic units (OTUs) and taxonomic classifications were determined by BLAST searching the sequences against the Greengenes database at the $97 \%$ nucleotide similarity level [19]. The $\alpha$ diversity was represented according to the Shannon, Simpson, ACE, and Chao indexes. The taxonomybased analyses were visualized based on GraPhlAn. The data were visualized via principal component analysis (PCA) [20].

2.4. Statistical Analysis. All data are expressed as the mean \pm SD and were subjected to one-way analysis of variance (ANOVA), Fisher's least significant difference (LSD) test, or Spearman's correlation test using SPSS 23.0 (SPSS Inc., IBM, USA). The PCA score plots based on phylogenetic statistical analysis methods indicated clustering of the gut microbiota within groups. The Wilcoxon rank-sum test was used to compare the group data of two samples. $P<0.05$ was considered statistically significant. The results were graphically visualized using GraphPad Prism (v6.0, GraphPad Software, USA), and correlation heatmaps were generated using $R$ software (v3.3.1).

\section{Results}

In a preliminary experiment, it was observed that the mice began to die $10 \mathrm{~h}$ after D-Gal $(0.5 \mathrm{~g} / \mathrm{kg}) / \mathrm{LPS}$ injection; thus, to ensure that no mice died during the experiment, we chose to perform euthanasia at $8 \mathrm{~h}$.

3.1. Mouse Body Weight. There was a gradual increase in body weight in all groups. In week 2 , the body weights of the mice were not significantly increased in any of the four groups (Figure 1).

3.2. ALF Attenuation. The liver weight of the mice did not significantly differ among the four groups (Figure 2). The livers of the control group mice had a light-red smooth surface and a soft texture. In contrast, D-Gal/LPS treatment caused severe liver failure. The livers of the Mod mice were dark red and exhibited coagulation, necrosis, and a slightly hard texture. Sil and Xfd reduced the ALF changes, according to visual observations (Figure 3). The serum ALT and AST levels in the Mod mice were much higher than those in the Con mice. Compared with the Mod group, the Sil and Xfd groups showed remarkably reduced ALT and AST levels, and the Xfd mice showed no significant decreases in ALT and AST levels compared with those in the Sil mice (Figure 4).

The livers of the Con mice had clear liver lobules, and no degeneration or necrosis of liver cells was observed. In contrast, D-Gal/LPS treatment caused severe liver failure. The livers of the Mod mice had a large amount of hepatocyte degeneration, mainly cellular edema. Nuclear lysis, necrosis,

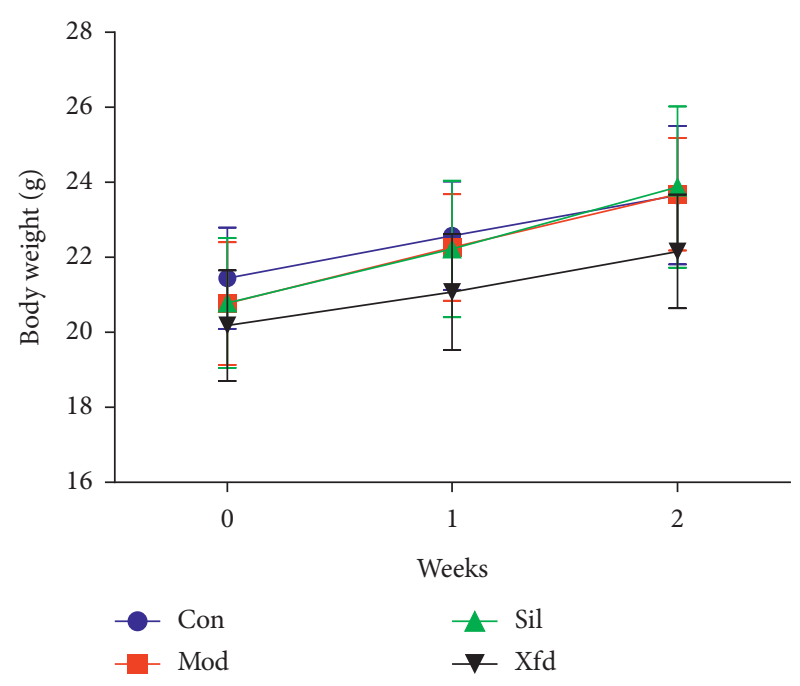

FIgURE 1: Comparison of body weights.

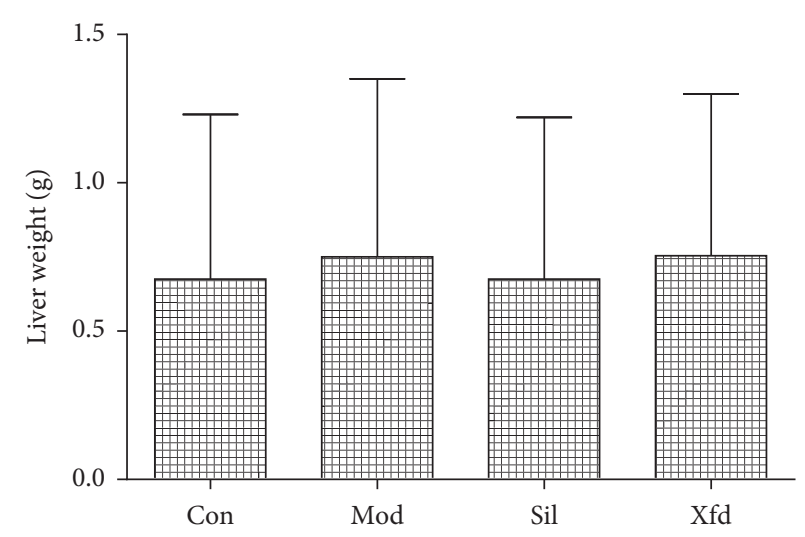

FIgURE 2: Comparison of liver weights and appearance.

inflammatory cell infiltration, massive bleeding, venous congestion and expansion, and no cell proliferation were observed. Compared with the Mod group, the Sil and Xfd groups showed reduced hepatocyte necrosis, bleeding, and inflammation, and the ALF changes were significantly improved (Figure 5).

3.3. Community Structure of the Gut Microbiota. To characterize the composition of the gut microbiota, 1,542,312 cleaned sequences were generated from 32 samples. In total, $567,608,501$, and 540 OTUs were found in the Con, Mod, Sil, and Xfd groups, respectively. In total, 747 operational OTUs were obtained. Moreover, there were 364 common OTUs across the four groups, and 16, 47, 19, and 41 specific OTUs were found in the Con, Mod, Sil, and Xfd groups, respectively (Figure 6).

The Shannon, Simpson, ACE, and Chao indexes were analyzed as $\alpha$ diversity metrics. There was an obvious increase in the ACE- and Chao-based diversity $(P<0.05)$ following Sil or Xfd treatment (Figure 7). There were no observed differences in the Shannon $(P=0.055)$ and Simpson $(P=0.217)$ indexes. 


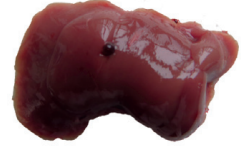

Con

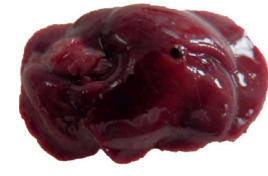

Mob

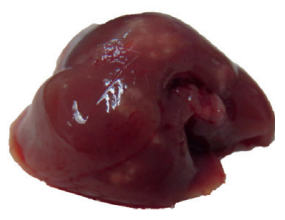

Sil

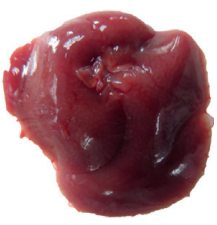

Xfd

(d)

Figure 3: Representative gross liver appearance. (a) Con. (b) Mod. (c) Sil. (d) Xfd.

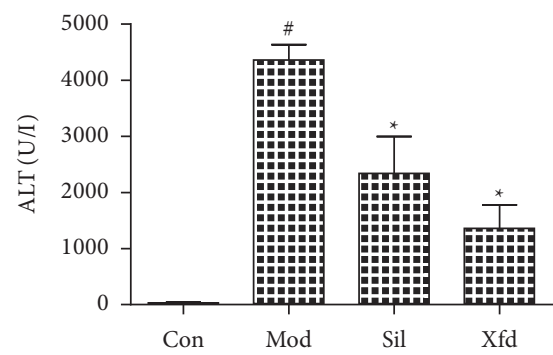

(a)

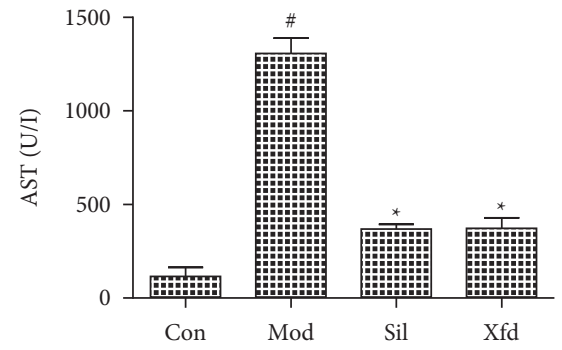

(b)

Figure 4: Comparison of ALT and AST levels (a) ALT. (b) AST. Note. The values are expressed as the mean \pm SD. ${ }^{\#} P<0.01$, compared with Con; ${ }^{\star} P<0.01$, compared with Mod.

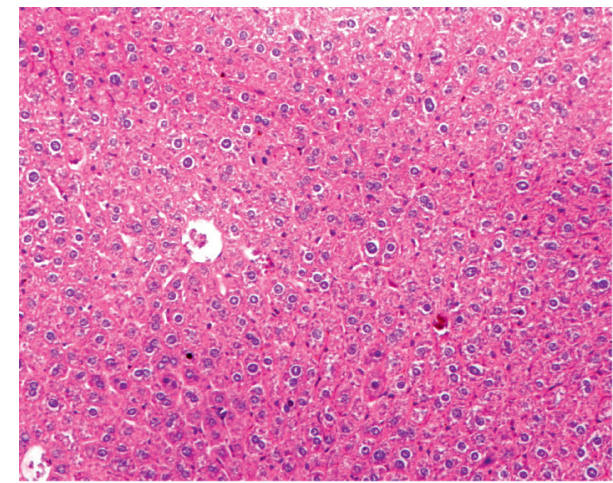

(a)

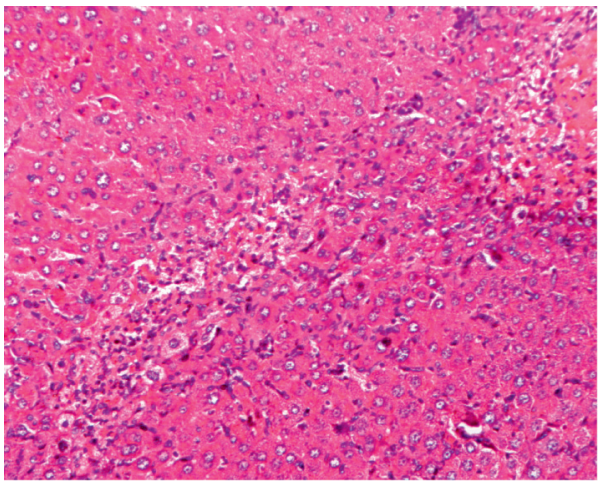

(c)

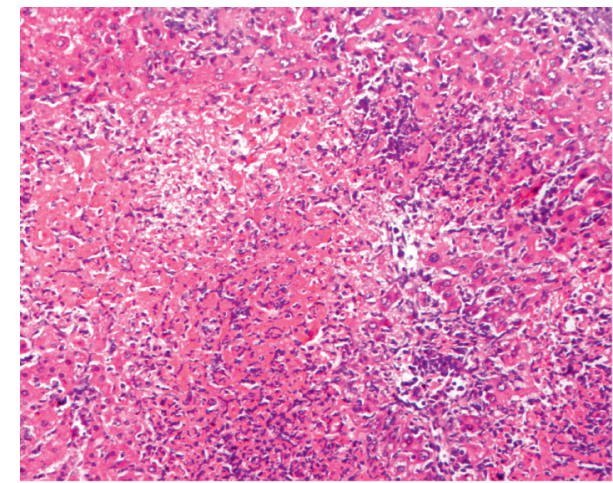

(b)

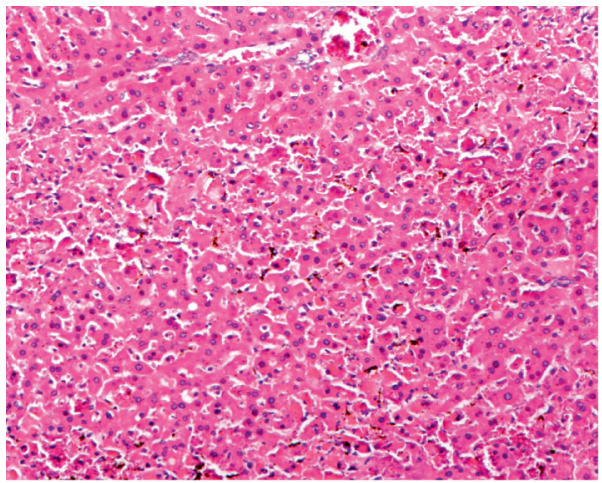

(d)

Figure 5: Representative histopathological changes in the liver. Note. Original magnification, 200×. (a) Con group. (b) Mod group. (c) Sil group. (d) Xfd group. 


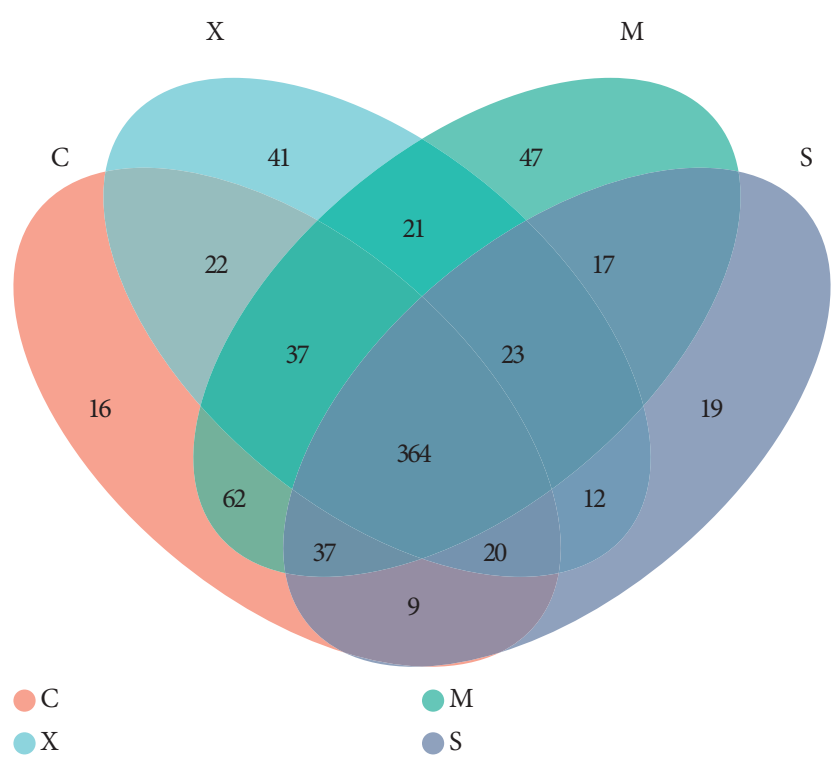

Figure 6: Comparison of OTUs.

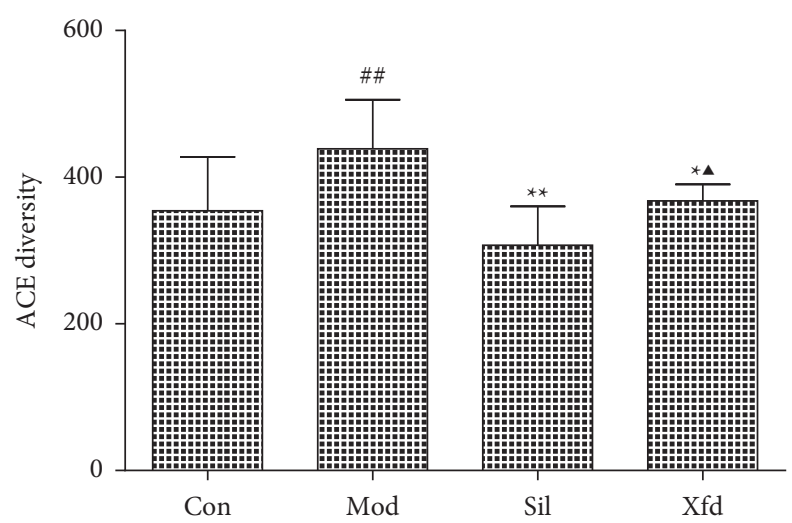

Figure 7: Comparison of the ACE diversity index. Note. The values are expressed as the mean $\pm \mathrm{SD}$; ${ }^{\#} P<0.01$, compared with the Con group; ${ }^{\star} P<0.05$ and ${ }^{\star \star} P<0.05$, compared with the Mod group; ${ }^{\star} P<0.05$, compared with the Sil group; C: Con group; M: Mod group; S: Sil group; X:Xfd group.

The PCA of both unweighted and weighted UniFrac distances showed that the first principal component (PC1) accounted for $71.68 \%$ and the second principal component (PC2) accounted for $16 \%$ of the variation. PCA also showed a reversal of the changes that occurred in the gut microbiota in response to the Sil and Xfd treatments, especially an obvious shift along PC1 (Figures 8 and 9).

3.4. Modulation of the Key Gut Microbiota Community Members. The dominant gut bacteria belonged to the phyla Firmicutes and Bacteroidetes. The composition of each sample is shown in detail (Figure 10). The abundance of Firmicutes in the Mod group $(45.85 \pm 13.00 \%)$ was significantly lower than that in the Con group $(70.67 \pm 15.34 \%)$ $(P<0.01)$. The proportion of Firmicutes showed an increasing trend in the Sil and Xfd groups $(72.30 \pm 14.30 \%$, $65.37 \pm 7.74 \%)$ compared with that in the Mod groups $(P<0.01, P<0.05)$. The abundance of Bacteroidetes in the
Mod group (34.62 $\pm 21.23 \%)$ was significantly higher than that in the Con group $(19.95 \pm 13.92 \%)(P<0.05)$. The proportion of Bacteroidetes showed a decreasing trend in the Sil and Xfd groups $(5.90 \pm 4.88 \%, 18.68 \pm 9.71 \%)$ compared with that in the Mod group $(P<0.01, P<0.05)$. The gut microbiota of the Mod group mice was characterized by an increased Bacteroidetes/Firmicutes ratio $(0.90 \pm 0.71)$, and the ratio showed significant differences, with lower proportions in the Sil and Xfd group mice $(0.08 \pm 0.07$, $0.30 \pm 0.19$, respectively) $(P<0.05, P<0.01)$. There was no statistically significant difference in the abundances of Firmicutes and Bacteroidetes or the Bacteroidetes/Firmicutes ratio between the Sil and Xfd groups $(P<0.05)$. There were no significant differences in the Actinobacteriota and Desulfobacterota abundances among the four groups (Figures 10-12).

At the genus level, the microbiota composition greatly varied among the four groups (Figure 13). Compared with those in the Mod group, higher proportions of 


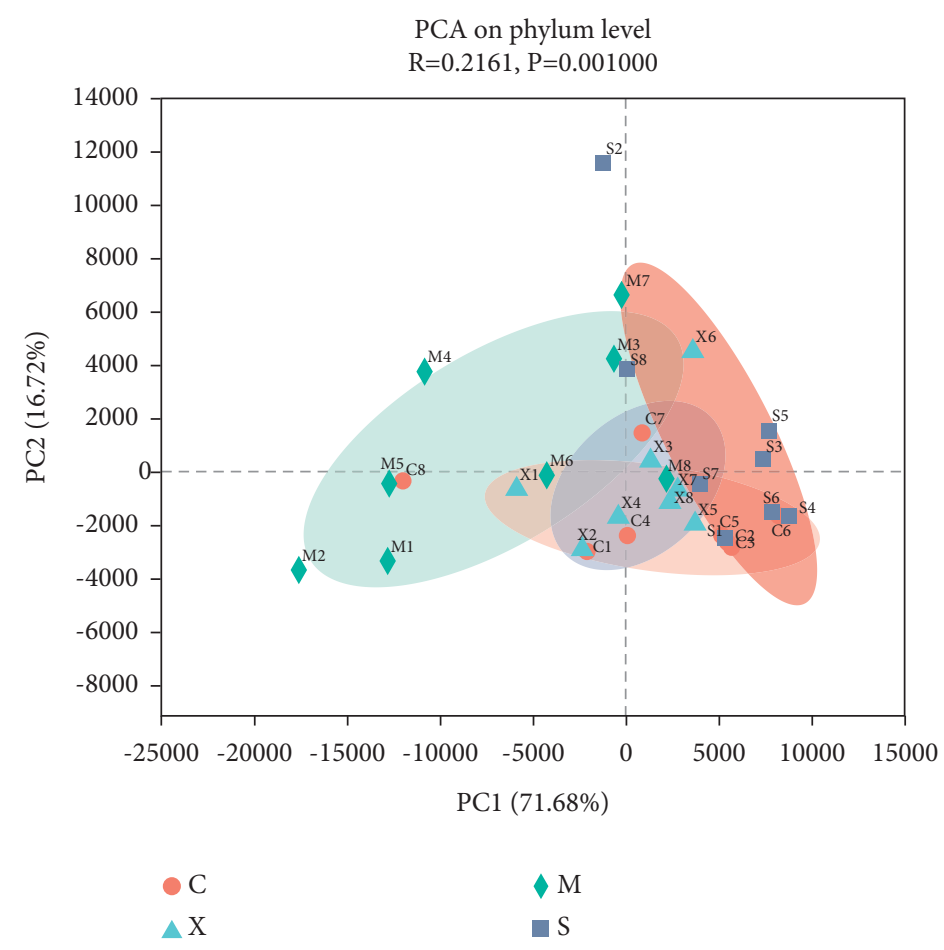

FIGURE 8: Comparison of the gut microbiota structure using PCA. Note. Different colors represent sample groups in different environments; C: Con group; M: Mod group; S: Sil group; X:Xfd group.

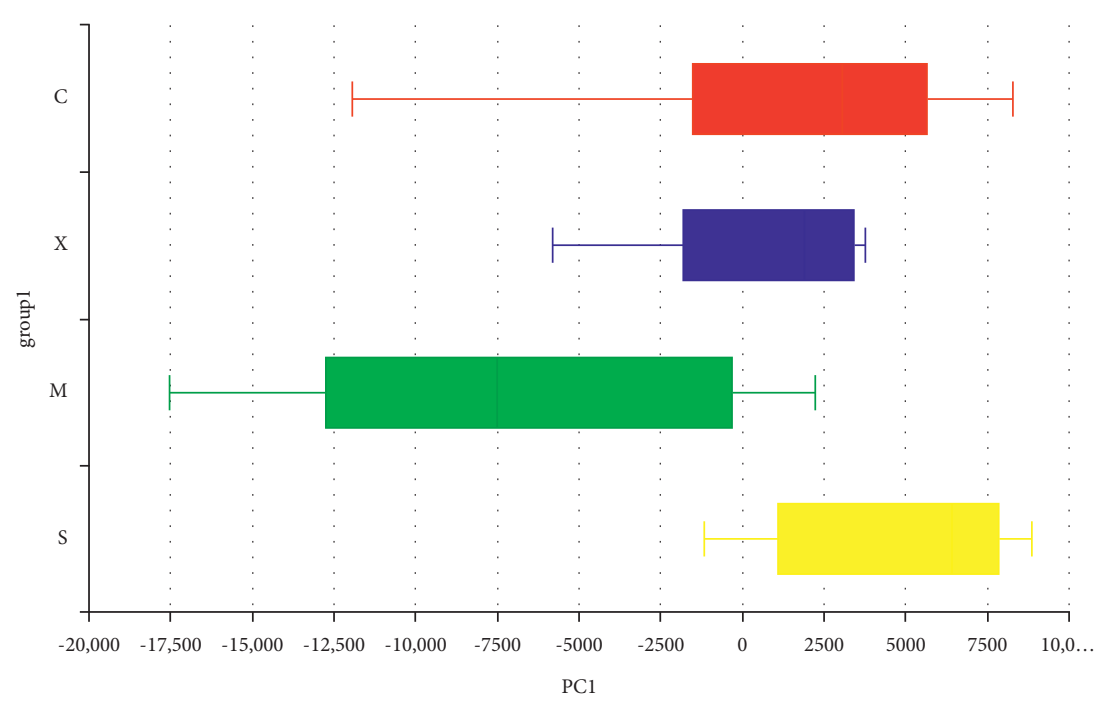

FIGURE 9: Comparison of the gut microbiota structure along PC1 using PCA. Note. The box plot in the figure represents the dispersion of different groups of samples on the PC1 axis; C: Con group; M: Mod group; S: Sil group; X:Xfd group.

Lachnospiraceae_NK4A136_group and Candidatus_Saccharimoas were observed in the Sil and Xfd groups $(P<0.01, \quad P<0.05)$. Otherwise, compared with the Sil treatment, the Xfd treatment did not induce remarkable increases in abundance $(P<0.05)$ (Figures 14 and 15). A reduction in Muribaculaceae abundance was detected in the Sil group compared with that in the Mod and Xfd groups $(P<0.01)$. Xfd treatment did not induce a significantly reduced relative abundance compared to that in the Mod group $(P<0.01)$ (Figures 14-16).

3.5. Correlations between ALF-Related Factors and the Gut Microbiota. A correlation heatmap analysis was performed to investigate ALF-related factors and the significantly affected gut microflora at the genus level. We found that the 


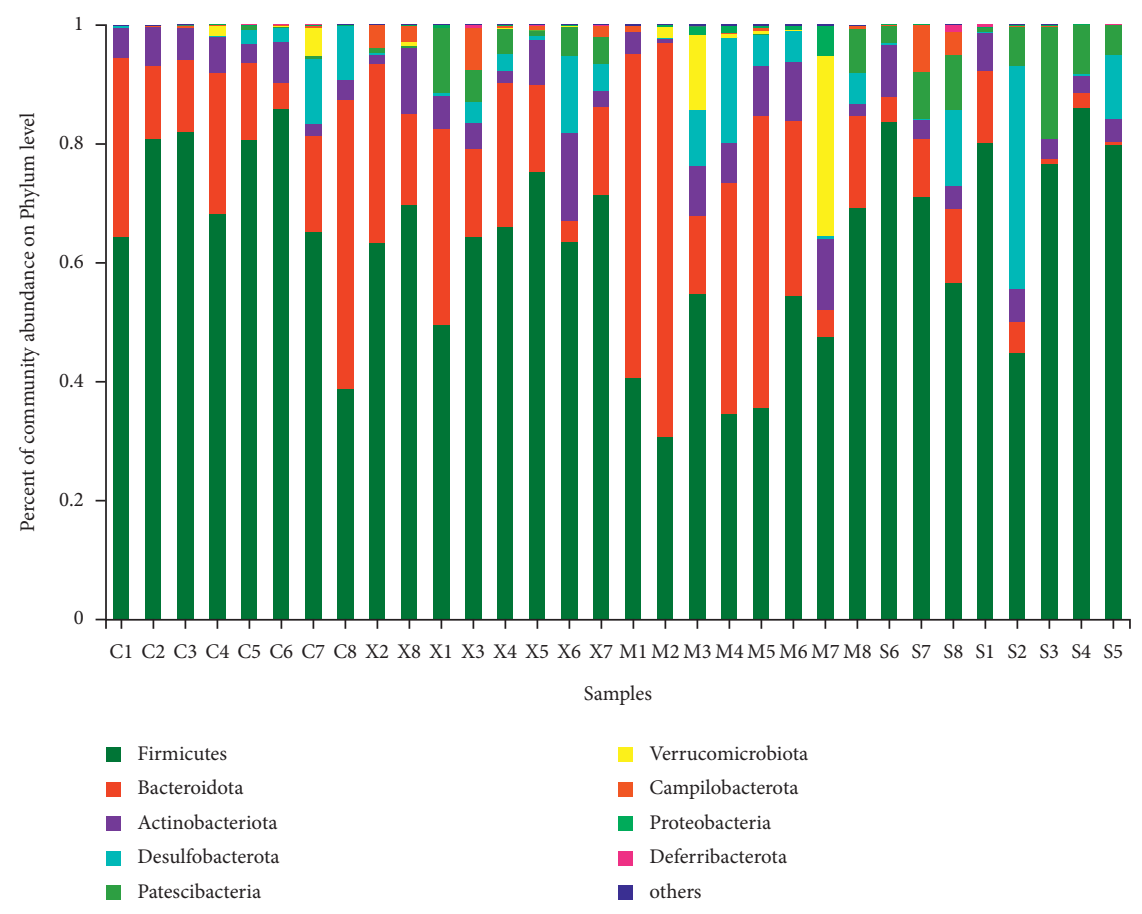

FIGURE 10: Comparison of the abundances of gut bacterial phyla.

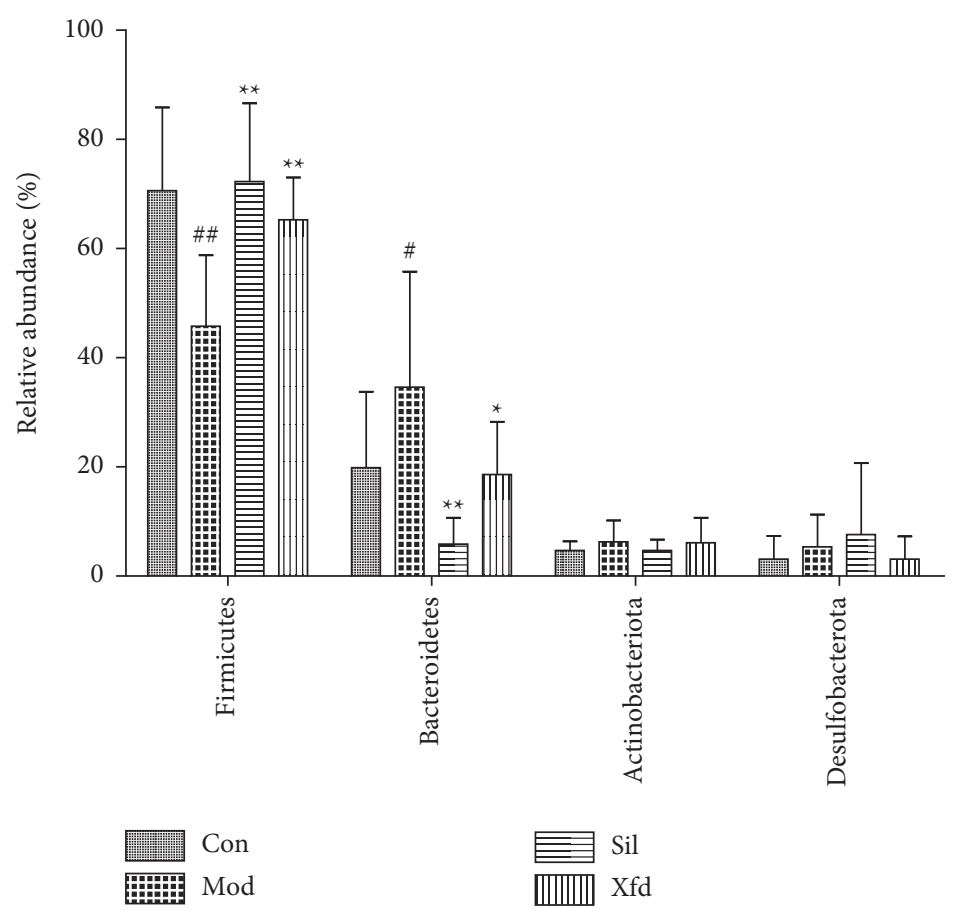

FIGURE 11: Comparison of the relative abundances of gut bacterial phyla. Note. $\# \# P<0.01$ versus the Con group; ${ }^{\star} P<0.05$ and ${ }^{\star \star} P<0.01$ versus the Mod group. 


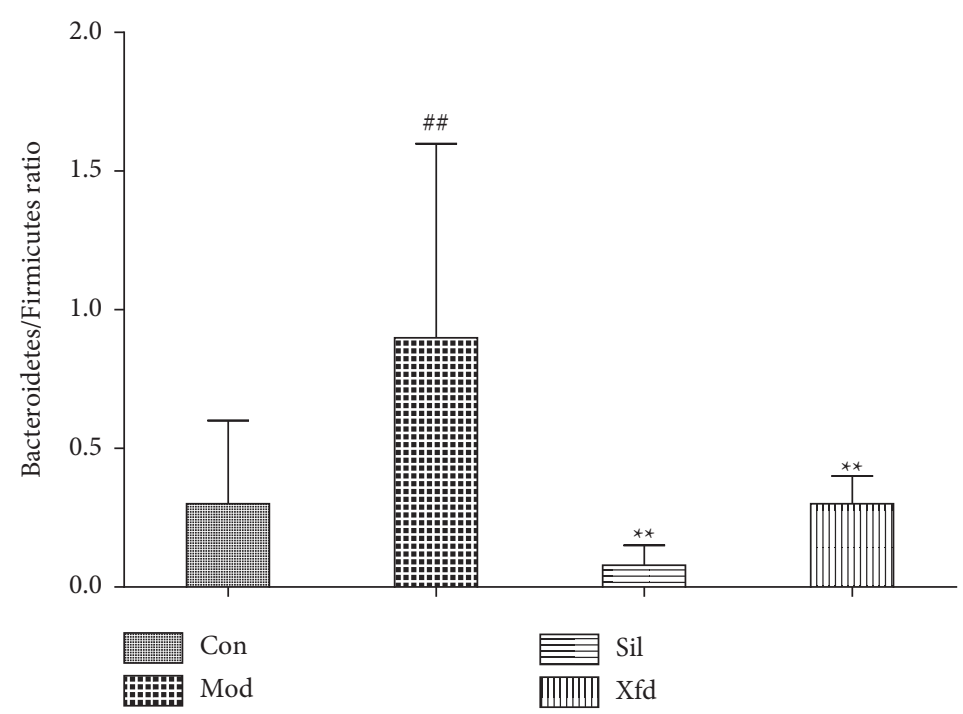

Figure 12: Comparison of the Bacteroidetes/Firmicutes ratio. Note. ${ }^{\# \#} P<0.01$ versus the Con group; ${ }^{\star \star} P<0.01$ versus the Mod group.
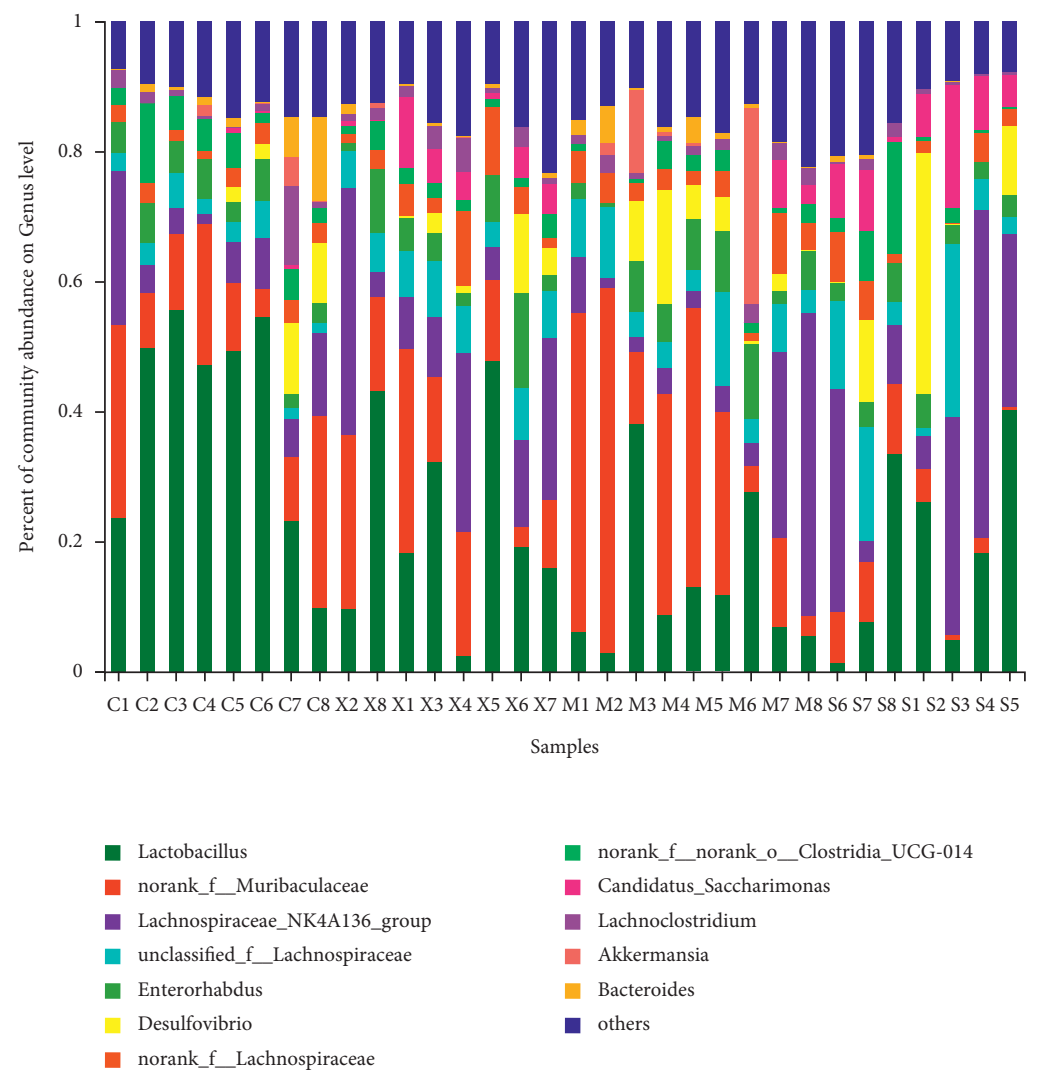

- norank_f_norank_o__Clostridia_UCG-014

- Candidatus_Saccharimonas

- Lachnoclostridium

- Akkermansia

- Bacteroides

others

FIGURE 13: Comparison of abundances among gut bacterial genera. 

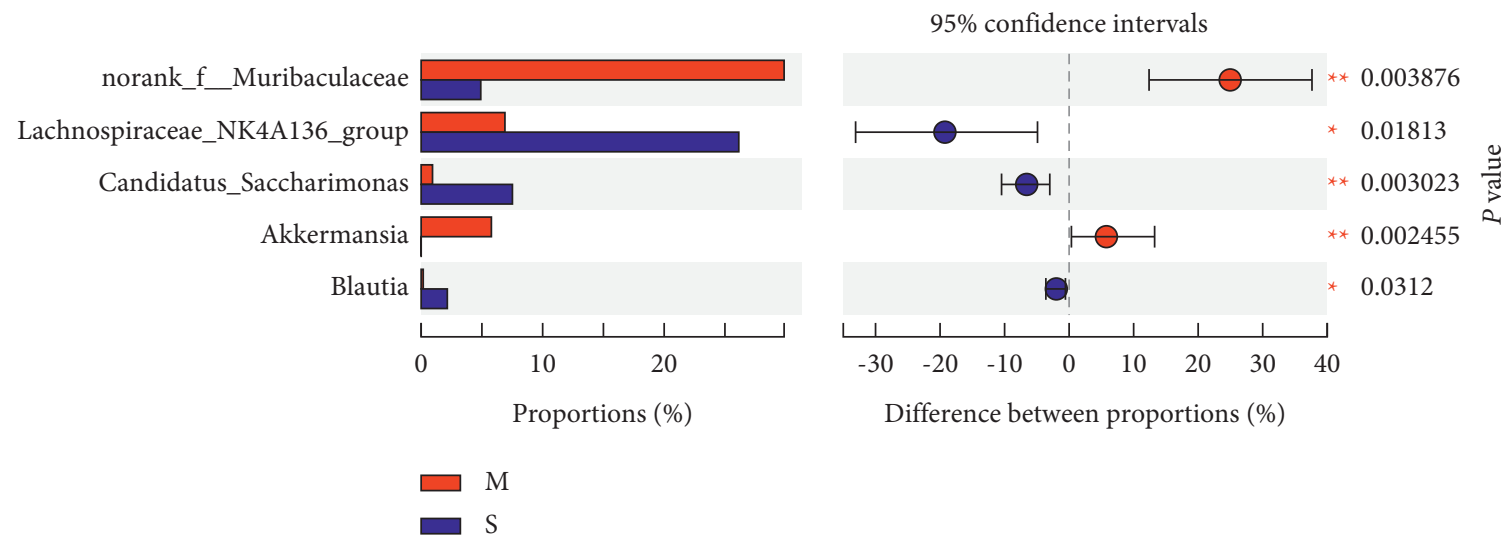

Figure 14: Comparison of the relative abundances of gut bacterial genera between the Mod and Sil groups. Note. ${ }^{\star} P<0.05$ and ${ }^{\star \star} P<0.01$ versus the Mod group; M: Mod group; S: Sil group.

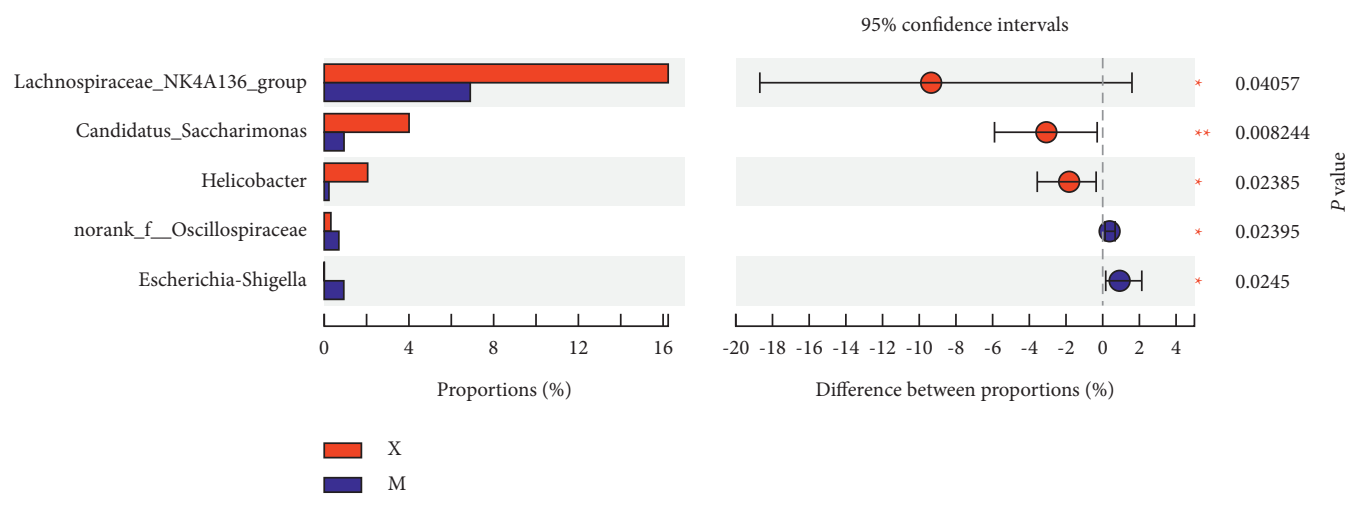

FIgURE 15: Comparison of the relative abundances of gut bacterial genera between the Mod and Xfd groups. Note. ${ }^{\star} P<0.05$ and ${ }^{\star \star} P<0.01$ versus the Mod group; M: Mod group; X: Xfd group.

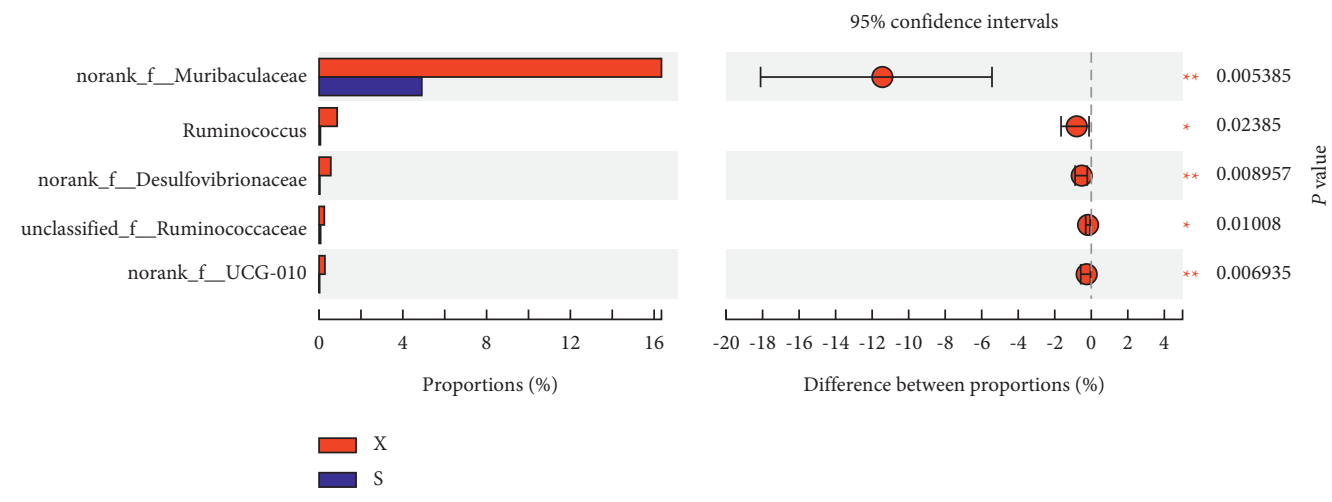

Figure 16: Comparison of the relative abundances of gut bacterial genera between the Sil and Xfd groups. Note. ${ }^{\star} P<0.05$ and ${ }^{\star \star} P<0.01$ versus the Sil group; S: Sil group; X: Xfd group.

ALT and AST levels were negatively correlated with Clostridia_UCG-014 abundance $\quad(P<0.05$ and $P<0.01)$ (Figure 17).

\section{Discussion}

ALF is a devastating clinical syndrome with high mortality and a substantial disease burden [21]. Although liver transplantation is the best treatment strategy for ALF, cadaveric organ shortages and subsequent complications limit this possibility. Multiple organ support and the use of antivirals and cell-based therapies demonstrate less encouraging efficacy; thus, great labor, material, and capital resources have been expended to identify alternative treatment methods for ALF in China [22].

Massive liver cell necrosis and extensive inflammation are the major changes in ALF [23, 24]. D-Gal/LPS can greatly stimulate inflammatory responses and increase 


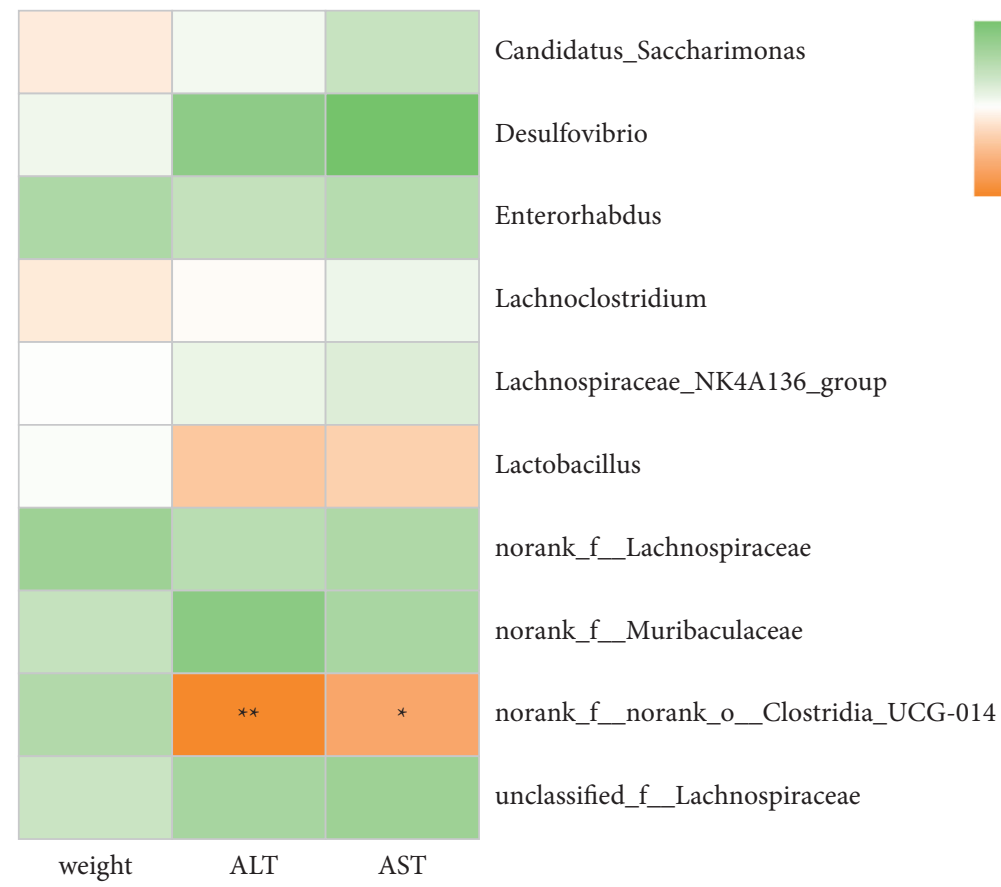

FIGURE 17: The relationship between ALF-related biochemical factors and the gut microbiota at the genus level. Note. The colors range from yellow (negative correlation) to green (positive correlation); statistical significance was based on ${ }^{\star} P$ value $<0.05$ or ${ }^{\star \star} P$ value $<0.01$.

endotoxin sensitivity and death in liver cells. An ALF model has been well established $[14,25]$. From the perspective of TCM, "stasis" and "phlegm" define the key pathogenic features of ALF [26]. We used Xfd, which is a prescription that has been applied to treat ALF. Xfd regulates the liver, promotes blood circulation, strengthens the spleen, and resolves phlegm $[27,28]$.

$\mathrm{BALB} / \mathrm{c}$ mice are widely used in animal immunology experiments. Since the weight change in the mice after 2 weeks was not very obvious, a significant difference was not observed. Moreover, Xfd had no effect on the weight of the liver. ALT and AST are major biochemical indicators of liver function. The ALT and AST levels in the Mod group were significantly increased compared with those in the Con group. Additionally, these elevations were rescued after Sil and Xfd pretreatment, indicating protective effects on ALF. The major histopathological changes in the livers of the mice after D-Gal/LPS exposure were the development of necrotic areas and severe hepatocyte necrosis. The liver appearance and texture associated with ALF were improved. Moreover, the Sil and Xfd treatments could also protect the liver tissue microstructure. The improvement in ALT and AST levels may be the most important reason.

These data clearly reveal that D-Gal/LPS-induced ALF was attenuated by Xfd treatment. We did not perform a high-performance liquid chromatography analysis of Xfd. This is a shortcoming of this study. We will perform this analysis in future research to obtain a clearer understanding of the main compounds present in Xfd. Tanshinone IIA is the primary active component extracted from Salviae Miltiorrhizae Radix et Rhizoma. Ma [29] reported pathological effects, revealing that tanshinone IIA remarkably mitigated pathologic damage to the liver, reducing hemorrhage and inflammatory cell infiltration. Consistent with the pathology results, tanshinone IIA improved ALT levels. Wang [30] demonstrated that tanshinone IIA pretreatment could protect the liver by activating the Nrf2 pathway. Schisandrae Fructus Chinensis prevented elevations in serum biochemical parameters, including AST and ALT levels. The mechanisms of Schisandrae Fructus Chinensis against druginduced liver damage involve the regulation of inflammatory factors and oxidative stress [31]. Studies have suggested that Schisandrae Fructus Chinensis can accelerate the repair and regeneration of liver cells. In this experiment, this phenomenon was not observed due to the short time span from establishing the model to killing the mice. Saikosaponins, which are major bioactive compounds in Bupleuri Radix, possess anti-inflammatory and antiviral activities, regulate cell apoptosis, and have hepatoprotective effects, as described in a comprehensive review [32]. Furthermore, increased risks of overdose-induced histological injury in the mouse liver have also been reported and were involved in saikosaponin D-induced mitochondrial apoptosis in liver cells and potential hepatotoxicity [33]. Citri Reticulatae Pericarpium was shown to inhibit adipogenesis in 3T3-L1 preadipocytes. A marked reduction in PPAR- $\gamma$, C/EBP- $\alpha$, and SREBP-1 levels played a positive role in protection against ALF [34]. Mume Fructus might play a therapeutic role in the improvement in ALT and AST levels by enhancing superoxide dismutase (SOD) activity and lowering malondialdehyde (MDA) content [35]. Accordingly, oxidative stress was improved in liver tissues, and hepatoprotective activities were observed [36].

Accumulating evidence indicates that multiple pathogenic factors that accelerate ALF are associated with gut microbiome imbalance [37-39]. Altered gut microbiota in 
terms of OTUs, the ACE diversity index, and PCA has been described by different groups. At the phylum level, Firmicutes and Bacteroidetes were the two main phyla of bacteria in the gut microbiota, and we found that the abundances of these two phyla were altered during D-Gal/LPS-induced ALF. An increased abundance of Firmicutes and a decreased abundance of Bacteroidetes have been widely reported to be associated with liver failure $[40,41]$. There are conflicting conclusions regarding the abundances of Bacteroidetes and Firmicutes. For example, Yan and $\mathrm{Hu}$ reported that consumption of a high-fat diet caused an increased abundance of Bacteroidetes and a decreased abundance of Firmicutes $[42,43]$. The abundance of Firmicutes was distinctly increased, and the abundance of Bacteroidetes and the Bacteroidetes/Firmicutes ratio were significantly decreased following Sil and Xfd treatment. However, the differences in Actinobacteriota and Desulfobacterota abundances were not significant due to the insufficient sample size. The fact that Xfd treatment reversed the gut microbiome imbalance may explain its hepatoprotective effects.

At the genus level, the microbiota composition greatly varied among the four groups. Currently, knowledge regarding the relationships between the members of Muribaculaceae and ALF is limited. Our data revealed that the decreased Muribaculaceae abundance might be a protective effect of Sil treatment. However, the effect of Xfd was not very obvious. Consistent with our results, Wang $G$ found that walnut green husk polysaccharide consumption decreased the relative abundance of norank_f_Muribaculaceae at the genus level during a liver injury in Kunming male mice [44]. Polyene phosphatidylcholine is used to treat liver injury [45]. After polyene phosphatidylcholine administration, the relative abundance of Firmicutes also increased at the phylum level. Moreover, we found that the relative abundance of Lachnospiraceae_NK4A136_group was obviously elevated [46]. Surana demonstrated that an increased abundance of Lachnospiraceae_NK4A136_group was associated with anti-inflammatory effects [47]. Patients with ALF display evidence of systemic inflammatory response syndrome and local liver inflammation [48]. Huang provided evidence suggesting that the expression levels of IL$17 \alpha$ and TLR2 were negatively correlated with the abundance of Candidatus_Saccharimonas. In their research, Qingluo Tongbi decoction was shown to play a therapeutic role by decreasing the inflammatory responses regulated by the gut microbiota [49]. In our study, it was observed that the Sil and Xfd treatments can reduce the abundance of Candidatus_Saccharimonas, which may explain their protective effects against liver failure. We found a negative correlation between the abundance of Clostridia_UCG-014 and the levels of liver function biochemical indicators, especially ALT, suggesting the beneficial role of these bacteria in liver health. However, our knowledge regarding Clostridia_UCG-014 is very limited. Clostridia are thought to be short-chain fatty acid (SCFA) producers. SCFAs play an important role in human health based on the gut microbiota-host lipid metabolism axis [50,51]. We sought to further investigate whether SCFA producers enriched by Sil and Xfd treatment prevent the progression of liver damage, but due to the lack of stool samples, this hypothesis was impossible to explore.

In conclusion, our findings demonstrate that Sil and Xfd treatment could notably alleviate D-Gal/LPS-induced ALF in mice, which might be correlated with decreased ALT and AST levels and reduced liver pathological damage. These protective effects may be mediated by alteration of the gut microbiota in ALF. Our findings provide new insight into the pretreatment of ALF and suggest that Xfd has probiotic potential against ALF and that its hepatoprotective effect on ALF patients needs to be proven in future clinical trials.

\section{Data Availability}

The data used to support the findings of this study are included within the article.

\section{Conflicts of Interest}

The authors declare that they have no conflicts of interest.

\section{Authors' Contributions}

Jindong Zhao, Zhaohui Fang, Yan Li, and Guoliang Zhang participated in the design of the study and wrote the manuscript. Lili Liu, Yunxia Lu, Xiaojun Yang, Yong Hou, Hao Zhou, and Yonghua Liu performed the experiments. Ling Xin, Mei Shi, and Sha Han helped complete the data analysis.

\section{Acknowledgments}

The current study was supported by the University Scientific Research Projects of Anhui (KJ2019A0442 and KJ2020A0401), State Administration of Traditional Chinese Medicine Xu Jingshi Master of Traditional Chinese Medicine Inheritance Studio (2015-24), Anhui University of Chinese Medicine Project (2021-zlgcfy017).

\section{References}

[1] S. Blasco-Algora, J. Masegosa-Ataz, M. L. Gutiérrez-García, S. Alonso-López, and C. M. Fernández-Rodríguez, "Acuteon-chronic liver failure: pathogenesis, prognostic factors and management," World Journal of Gastroenterology, vol. 21, no. 42, pp. 12125-12140, 2015.

[2] S. Celaj, M. W. Gleeson, J. Deng et al., "The microbiota regulates susceptibility to Fas-mediated acute hepatic injury," Laboratory Investigation, vol. 94, no. 9, pp. 938-949, 2014.

[3] C. Duan, Y. Zhao, C. Huang et al., "Hepatoprotective effects of Lactobacillus plantarum C88 on LPS/D-GalN-induced acute liver injury in mice," Journal of Functional Foods, vol. 43, no. 4, pp. 146-153, 2018.

[4] W. M. Lee and E. Seremba, "Etiologies of acute liver failure," Current Opinion in Critical Care, vol. 14, no. 2, pp. 198-201, 2008.

[5] A. Alam, K. Chun Suen, and D. Ma, “Acute-on-chronic liver failure: recent update," Journal of Biomedical Research, vol. 31, no. 3, pp. 283-300, 2017.

[6] W. Lee, "Acute liver failure," Seminars in Respiratory and Critical Care Medicine, vol. 33, no. 1, pp. 36-45, 2012. 
[7] F. Liu, Z. Sun, P. Hu et al., "Determining the protective effects of Yin-Chen-Hao Tang against acute liver injury induced by carbon tetrachloride using 16S rRNA gene sequencing and LC/MS-based metabolomics," Journal of Pharmaceutical and Biomedical Analysis, vol. 174, no. 9, pp. 567-577, 2019.

[8] H. Qiu, D. Mao, N. Tang et al., "The underlying mechanisms of Jie-Du-Hua-Yu granule for protecting rat liver failure," Drug Design, Development and Therapy, vol. 13, no. 2, pp. 589-600, 2019.

[9] W. Yang, Y. Hao, W. Hou et al., "Jieduan-niwan formula reduces liver apoptosis in a rat model of acute-on-chronic liver failure by regulating the E2F1-mediated intrinsic apoptosis pathway," Evidence-Based Complementary and Alternative Medicine, vol. 2019, Article ID 8108503, 11 pages, 2019.

[10] Y. Wang, J. S. Xu, and Y. P. LI, "Brief analysis of the ' 32 words' liver regulating method proposed by traditional Chinese medicine master XU Jing-shi," Chinese Journal of Traditional Chinese Medicine and Pharmacy, vol. 33, no. 6, pp. 2395-2397, 2018.

[11] G. L. Zhang, Y. LI, L. Zhang et al., "Clinical observation of xiaohuafuning decoction in treatment of chronic atrophic gastritis with syndrome of liver-stagnation and spleen-deficiency," Liaoning Journal of Traditional Chinese Medicine, vol. 42, no. 5, pp. 981-983, 2015.

[12] G. L. Zhang, Y. Li, J. D. Zhao et al., "Effect of Xiaohua funing decoction on ERK1/2 and p-ERK1/2 in rats with liver stagnation and spleen deficiency-type chronic atrophic gastritis," Journal of Anhui University of Chinese Medicine, vol. 34, no. 22, pp. 64-67, 2015.

[13] W. Wang, L. Jiang, Y. Ren, M. Shen, and J. Xie, "Characterizations and hepatoprotective effect of polysaccharides from Mesona blumes against tetrachloride-induced acute liver injury in mice," International Journal of Biological Macromolecules, vol. 124, no. 11, pp. 788-795, 2019.

[14] C. Galanos, M. A. Freudenberg, and W. Reutter, "Galactosamine-induced sensitization to the lethal effects of endotoxin," Proceedings of the National Academy of Sciences, vol. 76, no. 11, pp. 5939-5943, 1979.

[15] M. K. Kemelo, L. Wojnarová, N. Kutinová Canová, and H. Farghali, "D-galactosamine/lipopolysaccharide-induced hepatotoxicity downregulates sirtuin 1 in rat liver: role of sirtuin 1 modulation in hepatoprotection," Physiological Research, vol. 63, no. 5, pp. 615-623, 2014.

[16] Chinese Pharmacopoeia Commission, The Pharmacopoeia of the People's Republic of China, vol. 1, China Medical Science and Technology Press, Beijing, China, 2020.

[17] R. C. Edgar, "UPARSE: highly accurate OTU sequences from microbial amplicon reads," Nature Methods, vol. 10, no. 10, pp. 996-998, 2013.

[18] J. B. Leikin and F. P. Paloucek, Clostridium perfringens Poisoning, Poisoning and Toxicology Handbook, pp. 892-893, Informa, 4th. edition, 2008.

[19] T. Z. DeSantis, P. Hugenholtz, N. Larsen et al., "Greengenes, a chimera-checked 16S rRNA gene database and workbench compatible with ARB," Applied and Environmental Microbiology, vol. 72, no. 7, pp. 5069-5072, 2006.

[20] A. Ramette, "Multivariate analyses in microbial ecology," Fems Microbiology Ecology, vol. 62, no. 2, pp. 142-160, 2007.

[21] N. Halliday and R. H. Westbrook, "Liver transplantation: need, indications, patient selection and pre-transplant care," British Journal of Hospital Medicine, vol. 78, no. 5, pp. 252259, 2017.
[22] R. Hernaez, E. Solà, R. Moreau, and P. Ginès, "Acute-onchronic liver failure: an update," Gut, vol. 66, no. 3, pp. 541-553, 2017.

[23] M. Bosmann, J. J. Grailer, R. Ruemmler et al., "Extracellular histones are essential effectors of C5aR- and C5L2-mediated tissue damage and inflammation in acute lung injury," The FASEB Journal, vol. 27, no. 12, pp. 5010-5021, 2013.

[24] R. Rani, A. Tandon, J. Wang, S. Kumar, and C. R. Gandhi, "Stellate cells orchestrate concanavalin A-induced acute liver damage," The American Journal of Pathology, vol. 187, no. 9, pp. 2008-2019, 2017.

[25] J. Yethon and C. Whitfield, "Lipopolysaccharide as a target for the development of novel therapeutics in gram-negative bacteria," Current Drug Target -Infectious Disorders, vol. 1, no. 2, pp. 91-106, 2001.

[26] L. Ding, X. Zhang, L. Li et al., "Qingchangligan formula alleviates acute liver injury by attenuating extracellular histoneassociated inflammation," Biomedicine and Pharmacotherapy, vol. 103, no. 1, pp. 140-146, 2018.

[27] Y. Li, J. D. Zhao, L. Xin et al., "Analysis on medication rules of professor XU Jingshi in treating stomachache of stagnation of liver qi stagnation and spleen deficiency type: based on data mining," Journal of Traditional Chinese Medicine, vol. 58, no. 15, pp. 1288-1292, 2017.

[28] L. L. Liu, G. L. Zhang, Y. H. Liu et al., "The relationship between TCM syndrome types and ALT, DNA HBV and liver tissue pathology in patients with chronic hepatitis B," World Chinese Medicine, vol. 12, no. 3, pp. 562-565, 2017.

[29] S. Ma, X. Wang, Y. Wang, and X. Zuo, "Sodium tanshinone IIA sulfonate improves hemodynamic parameters, cytokine release, and multi-organ damage in endotoxemia rabbits," Medical Science Monitor, vol. 24, no. 5, pp. 2975-2982, 2018.

[30] W. Wang, C. Guan, X. Sun et al., "Tanshinone IIA protects against acetaminophen-induced hepatotoxicity via activating the Nrf2 pathway," Phytomedicine, vol. 23, no. 6, pp. 589-596, 2016.

[31] X. K. Li, H. J. Yang, J. C. Xiao et al., "Network pharmacology based investigation into the bioactive compounds and molecular mechanisms of Schisandrae Chinensis Fructus against drug-induced liver injury," Bioorg Chem, vol. 96, Article ID 103553, 8 pages, 2020.

[32] X. Li, X. Li, N. Huang, R. Liu, and R. Sun, "A comprehensive review and perspectives on pharmacology and toxicology of saikosaponins," Phytomedicine, vol. 50, no. 9, pp. 73-87, 2018.

[33] F. Zhang, L. Chen, H. Jin et al., "Activation of Fas death receptor pathway and Bid in hepatocytes is involved in saikosaponin D induction of hepatotoxicity," Environmental Toxicology and Pharmacology, vol. 41, no. 1, pp. 8-13, 2016.

[34] F. Sheu, W. I. Chuang, and P. J. Chien, "Citri Reticulatae Pericarpium extract suppresses adipogenesis in 3T3-L1 preadipocytes," Journal of the Science of Food and Agriculture, vol. 87, no. 8, pp. 2382-2389, 2007.

[35] C. Chen, D.-C. Wen, S.-d. Gao, X.-Y. Hu, and C. Yi, "The protective effects of buzui on acute alcoholism in mice," Evidence-Based Complementary and Alternative Medicine, vol. 2016, Article ID 3539748, 8 pages, 2016.

[36] A. M. Soliman, H. M. Karam, M. H. Mekkawy et al., “Antioxidant activity of novel quinazolinones bearing sulfonamide: potential radiomodulatory effects on liver tissues via NF- $\kappa \mathrm{B} / \mathrm{PON} 1$ pathway," European Journal of Medicinal Chemistry, vol. 197, pp. 1-13, Article ID 112333, 2020.

[37] P. D. Cani, "Human gut microbiome: hopes, threats and promises," Gut, vol. 67, no. 9, pp. 1716-1725, 2018. 
[38] A. Tripathi, J. Debelius, D. A. Brenner et al., "The gut-liver axis and the intersection with the microbiome," Nature Reviews Gastroenterology and Hepatology, vol. 15, no. 7, pp. 397-411, 2018.

[39] C. A. Woodhouse, V. C. Patel, A. Singanayagam, and D. L. Shawcross, "Review article: the gut microbiome as a therapeutic target in the pathogenesis and treatment of chronic liver disease," Alimentary Pharmacology and Therapeutics, vol. 47, no. 2, pp. 192-202, 2018.

[40] N. Qin, F. Yang, A. Li et al., "Alterations of the human gut microbiome in liver cirrhosis," Nature, vol. 513, no. 7516, pp. 59-64, 2014.

[41] M. Mouzaki, E. M. Comelli, B. M. Arendt et al., "Intestinal microbiota in patients with nonalcoholic fatty liver disease," Hepatology, vol. 58, no. 1, pp. 120-127, 2013.

[42] H. Yan, J. Lu, Y. Wang, W. Gu, X. Yang, and J. Yu, "Intake of total saponins and polysaccharides from Polygonatum kingianum affects the gut microbiota in diabetic rats," Phytomedicine, vol. 26, no. 3, pp. 45-54, 2017.

[43] S. W. Hu, Y. L. Xu, X. Gao et al., "Long-chain bases from sea cucumber alleviate obesity by modulating gut microbiota," Marine Drugs, vol. 17, no. 8, pp. 455-464, 2019.

[44] G. Wang, Y. Zhang, R. Zhang et al., "The protective effects of walnut green husk polysaccharide on liver injury, vascular endothelial dysfunction and disorder of gut microbiota in high fructose-induced mice," International Journal of Biological Macromolecules, vol. 162, no. 6, pp. 92-106, 2020.

[45] M. Cao, X. Li, B. Zhang et al., "The effect of polyene phosphatidyl choline intervention on nonalcoholic steatohepatitis and related mechanism," American Journal of Translational Research, vol. 8, no. 5, pp. 2325-2330, 2016.

[46] H. Yu, Z. Yu, H. Huang et al., "Gut microbiota signatures and lipids metabolism profiles by exposure to polyene phosphatidylcholine," Biofactors, vol. 45, no. 3, pp. 439-449, 2019.

[47] N. K. Surana and D. L. Kasper, "Moving beyond microbiomewide associations to causal microbe identification," Nature, vol. 552, no. 7684, pp. 244-247, 2017.

[48] E. Triantafyllou, K. J. Woollard, M. J. W. McPhail et al., "The role of monocytes and macrophages in acute and acute-onchronic liver failure," Frontiers in Immunology, vol. 9, no. 10, pp. 1-17, 2018.

[49] Y. Huang, M. Li, L. Zhou et al., "Effects of Qingluo Tongbi decoction on gut flora of rats with adjuvant-induced arthritis and the underlying mechanism," Evidence-Based Complementary and Alternative Medicine, vol. 2019, Article ID 6308021, 11 pages, 2019.

[50] M. Kasubuchi, S. Hasegawa, T. Hiramatsu, A. Ichimura, and I. Kimura, "Dietary gut microbial metabolites, short-chain fatty acids, and host metabolic regulation," Nutrients, vol. 7, no. 4, pp. 2839-2849, 2015.

[51] J. D. Zhao, Y. Li, M. Sun et al., "Effect of berberine on hyperglycaemia and gut microbiota composition in type 2 diabetic Goto-Kakizaki rats," World Journal of Gastroenterology, vol. 27, no. 8, pp. 708-724, 2021. 